\title{
Parenting and Emotional and Behavioral Responses of Children and Adolescents to the COVID-19 Epidemic: A Cross-sectional Survey in China
}

Zhiyuan Hou ( $\nabla$ zyhou@fudan.edu.cn )

Fudan University

Fanxing Du

Fudan University

Li He

Beijing Normal University

Mark Francis

Tampere University

Mark Forshaw

Liverpool John Moores University

Kerry Woolfall

University of Liverpool

Qian Lv

Fudan University

Lu Shi

Clemson University

Hongjie Yu

Fudan University

\section{Research Article}

Keywords: children, mental health, emotion, behavioral response, COVID-19, China

Posted Date: January 15th, 2021

DOl: https://doi.org/10.21203/rs.3.rs-140407/v1

License: (1) (i) This work is licensed under a Creative Commons Attribution 4.0 International License.

Read Full License 


\section{Abstract}

To investigate parenting and children's emotional and lifestyle responses to the COVID-19 epidemic, we conducted an online survey of random, representative sample of residents with children aged 3-17 years in mid-March, 2020 in China. 1655 parents were surveyed with $80.1 \%$ response rate. During the epidemic, half (49\%) of children had stress symptoms and $10 \%$ had emotional problems; children had higher screen time, less exercise and worse sleep than before. Socially disadvantaged children were more vulnerable to the epidemic. Children whose parents communicated about the epidemic more frequently, who had irritable parents and experienced worse parent-child closeness had higher probabilities of emotional problems, stress symptoms and worse lifestyles. Improve parenting skills and communication quality is necessary during the epidemic.

\section{Introduction}

In late 2019, a novel coronavirus disease (COVID-19) emerged in Wuhan city, and spread rapidly across China and the world [1]. To control the transmission of COVID-19, the Chinese government implemented strict quarantine measures, and issued the highest-level public health alert in the country. A series of measures such as lockdown of cities, stay-at-home orders, closure of shops and schools, recommendations for delayed return to workplaces, as well as suspension of mass gatherings were implemented across the country [2]. The COVID-19 epidemic and consequent quarantine measures may have led to important psychological and lifestyle changes, which may have health consequences beyond the duration of the current epidemic. Chinese adults are reported to have longer periods of inactivity, increased screen time and more psychological health problems during the outbreak $[3,4]$. The nation-wide school closure confined more than 220 million children and adolescents to their homes [5]. Yet, whether and to what extent children's mental health and lifestyles have been affected during the COVID-19 epidemic in China is relatively unknown.

For children and adolescents, psychological well-being is often reflected by internalizing emotional and externalizing behavioral problems or disorders [6]. Previous studies have reported prevalent emotional or behavioral disorders and unhealthy lifestyles among children and adolescents before COVID-19. In China, approximately $13-24 \%$ of children are reported to have emotional or behavioral disorders $[7,8]$. If emotional and behavioral problems in children are not detected and treated as early as possible, serious disruptions in their educational progress, self-injury and development of other behavioral disorders are likely long-term outcomes [7]. According to World Health Organization (WHO) guidelines, children are recommended to keep physically active, have sufficient sleep and no more than 2 hours of screen time per day $[9,10]$. However, a national cross-sectional survey of 131,859 students aged $7-19$ years in China indicated that in 2019 , only $34.1 \%$ of children and adolescents had sufficient physical activity, and $65.4 \%$ adhered to the screen viewing guideline of two hours or less per day [11]. Since children and adolescents are vulnerable to public health emergencies, the epidemic and consequent series of quarantine measures may exacerbate emotional disorders and unhealthy lifestyles, and compound other existing socioeconomic inequities in health [12]. Rapid assessment of potential changes in the emotional health and 
lifestyle of children and adolescents during the epidemic can help detect early signs of emotional or behavioral problems and identify the children most at risk for later-life mental or physical disorders $[13,14]$.

Parents are known to play an important role in the establishment of a child's secure attachment relationships, for emotional support, self-regulation, and the development of lifestyle habits, according to the family systems theory [15]. With the implementation of household confinement, school closure and delays in parents' return to workplaces during the epidemic, social interactions have been limited to the closest family members [16]. During the epidemic, the increased interaction between parents and children may have changed their communication patterns, closeness and attitudes towards each other, which in turn can influence children's emotions and behaviors. It is important to quantify the influence of different parenting characteristics on children' emotional and lifestyle changes during the epidemic to determine how tailored interventions may be developed for parents and children during public health emergencies.

Our study aimed to 1) investigate changes in parenting and children's emotional and lifestyle responses to the COVID-19 epidemic, and potential inequities between socially disadvantaged and advantaged households; 2) examine associations between parenting characteristics and children's emotional and lifestyle responses to the epidemic. This study would help to detect the early signs of emotional and behavioral problems among children and adolescents, and develop interventions to deal with public health emergencies for parents and children in the Chinese context.

\section{Methods}

\subsection{Study design}

We conducted an online survey of random, representative sample of residents with children aged 317 years in Wuhan and Shanghai during March 12-17th, 2020. These two cities were selected to represent differing levels of challenges posed by the COVID-19 epidemic to parents and children. Wuhan is the epicenter of the COVID-19 epidemic and was under complete lockdown when the survey was conducted. Shanghai was significantly affected by imported COVID-19 cases from Wuhan and under partial lockdown when the survey was conducted. The COVID-19 cases reached around 5000 in Wuhan and 350 in Shanghai during our survey period. Wuhan was put in quarantine on January 23 , and ended its quarantine on April 8; Shanghai activated highest-level public health emergency response (PHER) on January 24, including stay-at-home orders, closure of shops and schools, as well as suspension of mass gatherings, and lifted it on March 24. Spring Festival national holiday started on January 24, and work and production resumed for parents on February 9 and March 10 in Shanghai and Wuhan, respectively. The schools reopened for children from early May, and online courses were taken during the school closure. Figure 1 illustrates the timeline of the epidemic progression and quarantine measures.

Our target population was children aged 3-17 years, who along with their parents resided in Wuhan or Shanghai for at least 6 months prior to the survey. The sample size ( $n=800$ for each city) was calculated 
to provide a sampling error of $3 \%$, and proportional quota sampling was done to ensure that respondents were demographically representative of the populations in each city, with quotas based on children's age and gender $[17,18]$. Respondents were sampled using the proportions of children in different age groups and by gender using estimates from a recent census [19], and survey sampling continued until the proportions of children in the age and gender quotas were achieved in Wuhan and Shanghai. Based on a national database with emails and household information registered, residents with children were randomly selected to receive the survey link, and informed consent was obtained at the beginning of the survey. Parents were asked to complete the questionnaire, and if they had a child aged 10 years or older, the child was asked to self-report their emotional responses and lifestyle (before and during the COVID-19 epidemic). In total, 2960 parents accepted the invitation to participate in the survey, and 2065 were eligible. Among them, 410 either did not complete the questionnaire or had missing data that disqualified their inclusion. Our final analytical sample consisted of 816 respondents in Wuhan and 839 respondents in Shanghai, and the effective response rate for the survey was $80.1 \%(1,655 / 2,065)$.

\subsection{Measures of parenting, and children's emotional responses and lifestyle changes during the COVID-19 epidemic}

The survey questionnaire is presented in the Appendix. Parenting was assessed by the frequency of parent-child communication about the COVID-19 epidemic (3 items), parent attitudes towards their child ( 1 item) and parent-child closeness ( 1 item) during the epidemic. Frequencies of children actively asking their parents about the epidemic, parents explaining about the epidemic to their children, and parents expressing negative sentiments or discussing the epidemic in the presence of their children were reported by parents to measure parent-child communication about the COVID-19 epidemic. Changes in the attitude of parents towards their children and parent-child closeness compared to the previous winter break were also reported by parents.

Children's internalized emotional problems (e.g., somatic complaints, depression, fear concerns) and externalized behavioral problems (e.g., aggressive behaviors) were enquired to assess their mental health during the epidemic. Emotional responses were measured using an Emotional Symptoms Score, which was based on the Strengths and Difficulties Questionnaire (SDQ) [20]. The tool is a brief, self-reported scale with demonstrated reliability and validity for use among parents and children [21]. Substantial risk of clinically significant emotional problems was defined as an SDQ-Emotional Symptoms Score $>4$ for parents or $>6$ for children and adolescents. Additionally, according to recommendations by the American Psychological Association, the frequency of externalized behavioral problems including 1) aggressive or stubborn behavior and 2) tantrums or meltdowns were used to measure the stress symptoms children experienced during the epidemic. Children who reported "never" to both the questions mentioned above were categorized as not having stress symptoms, and all the other responses were categorized as having stress problems. 
Children's lifestyles were measured and compared between two periods: (1) before the epidemic - the previous winter break - and (2) during the epidemic. The lifestyle aspects included: the frequency of going outside per week, screen time (hours per day), frequency of moderate to vigorous exercise (days per week and minutes per day), duration of sleep (hours per night) and sleep quality. We used the Sleep Disturbance Scale for Children [22] to measure children's sleep quality (i.e. difficulty getting to sleep and frequency of waking up at night).

\subsection{Statistical Analysis}

Descriptive analyses were used to investigate parenting, children's emotional responses and lifestyle changes during the COVID-19 epidemic. Chi-square tests or Fisher's exact tests (if expected frequency $<5$ ) were used to compare differences in these outcome measures between Wuhan and Shanghai, and before and during the epidemic (Tables 1 and 2). To measure changes in the lifestyle of children, we compared the proportion of children who had an unhealthy lifestyle during the previous winter break and during the COVID-19 epidemic. According to the World Health Organization recommendations on physical activity, sedentary behavior and sleep for good health $[9,10]$, children who (1) had a screen time of more than 2 hours per day, (2) exercised less than 3 times per week, (3) exercised less than 1 hour per day, (4) slept less than 8 hours per night, (5) had difficulty getting to sleep and (6) woke up more than twice per night were categorized as having an "unhealthy" lifestyle for the analyses (Fig. 2).

We also investigated potential inequities in parenting, emotional responses and lifestyle changes during the COVID-19 epidemic, between socially disadvantaged and advantaged families. The disadvantaged groups included children with parents who had low education, low family economic status, and who had not returned to work or were unemployed during the epidemic. For comparison, ratios were generated by dividing children's outcome proportions for the disadvantaged and advantaged groups, a ratio of 1 indicates no inequity between the groups (Table 3). P values were calculated using Chi-square tests or Fisher's exact tests (if expected frequency $<5$ ). In the equity analysis, children's lifestyle changes were measured as the proportions of children who had more screen time, less exercise, less sleep time, and more often had difficulty getting sleep or woke up more than twice per night during the epidemic than normally.

Multivariate logistic regressions were used to estimate the associations between parenting characteristics and children's emotional and lifestyle changes during the epidemic, controlling for different child characteristics (gender, age, fever symptoms or going outside during the epidemic), family characteristics (education, family economic status, employment status, having COVID-19 cases in neighborhood), respondent type, and location of residence (Table 4). Proportions, adjusted odds ratios (AOR) and 95\% confidence intervals $(\mathrm{Cl})$ are reported. All statistical analyses were performed using Stata 14.0 (Stata Corp LP, College Station, TX).

\section{Results}




\subsection{Participants' characteristics}

Table 1 shows that the characteristics of children in Wuhan and Shanghai were generally similar in terms of gender, age distribution and whether they experienced fever or cough symptoms during the epidemic. Characteristics of parents in Wuhan and Shanghai were similar in terms of household size and education, but a greater proportion of respondents in Wuhan reported confirmed or suspected COVID-19 cases in their neighborhood, had not returned to work and had a higher family economic status than in Shanghai. 
Table 1

Socio-demographic characteristics of participants in Wuhan and Shanghai, $\mathrm{n}(\%)$

\section{Characteristics}

Total

$(\mathrm{n}=$

1655)
Wuhan

$(n=$

816)
Shanghai $P$

$(n=839) \quad$ value*

Characteristics of children

Gender of children

0.751

Male

Female

Age of children, years

3-5

6-9

$10-14$

$15-17$

(26.

321

(19.4)

830

(50.2)

406

(49.8)

424

(50.5)

825

(49.9)

410

(50.3)

415

(49.5)

432

(26.1)

217

160

(19.6)

$32 \quad 217 \quad 215$

359

(21.7)

180

(22.1)

\section{3}

(32.8)

259

(31.7)

Children experienced fever symptoms during the epidemic

Yes

118

(7.1)

No

1537

(92.9)

760

$56(6.9)$

Family characteristics

Household size, mean (Standard Deviation)

3.5

(1.30)

3.5

$(1.26)$

Education level of parent

Three years college or below

Bachelor or above

Family economic status
161

(19.2)

179

(21.3)

284

(33.9)

62 (7.4)

0.838

(33.9)

0.677

777

(92.6)

.




\begin{tabular}{|c|c|c|c|c|}
\hline Characteristics & $\begin{array}{l}\text { Total } \\
(n= \\
1655)\end{array}$ & $\begin{array}{l}\text { Wuhan } \\
(n= \\
816)\end{array}$ & $\begin{array}{l}\text { Shanghai } \\
(n=839)\end{array}$ & $\begin{array}{l}P \\
\text { value* }\end{array}$ \\
\hline Low & $\begin{array}{l}188 \\
(11.4)\end{array}$ & $79(9.7)$ & $\begin{array}{l}109 \\
(13.0)\end{array}$ & \\
\hline Middle & $\begin{array}{l}886 \\
(53.5)\end{array}$ & $\begin{array}{l}410 \\
(50.3)\end{array}$ & $\begin{array}{l}476 \\
(56.7)\end{array}$ & \\
\hline High & $\begin{array}{l}581 \\
(35.1)\end{array}$ & $\begin{array}{l}327 \\
(40.1)\end{array}$ & $\begin{array}{l}254 \\
(30.3)\end{array}$ & \\
\hline Employment status & & & & $\begin{array}{l}<.001 \\
0.0\end{array}$ \\
\hline Work at office & $\begin{array}{l}644 \\
(38.9)\end{array}$ & $\begin{array}{l}273 \\
(33.5)\end{array}$ & $\begin{array}{l}371 \\
(44.2)\end{array}$ & \\
\hline Work at home & $\begin{array}{l}637 \\
(38.5)\end{array}$ & $\begin{array}{l}311 \\
(38.1)\end{array}$ & $\begin{array}{l}326 \\
(38.9)\end{array}$ & \\
\hline Have not returned to work or unemployed & $\begin{array}{l}374 \\
(22.6)\end{array}$ & $\begin{array}{l}232 \\
(28.4)\end{array}$ & $\begin{array}{l}142 \\
(16.9)\end{array}$ & \\
\hline COVID-19 cases in neighbourhood & & & & $<.001$ \\
\hline Yes & $\begin{array}{l}365 \\
(22.0)\end{array}$ & $\begin{array}{l}277 \\
(33.9)\end{array}$ & $88(10.5)$ & \\
\hline No or unclear & $\begin{array}{l}1290 \\
(78.0)\end{array}$ & $\begin{array}{l}539 \\
(66.1)\end{array}$ & $\begin{array}{l}751 \\
(89.5)\end{array}$ & \\
\hline \multicolumn{5}{|l|}{ Respondent } \\
\hline Respondent for overall questionnaire & & & & 0.099 \\
\hline Mother & $\begin{array}{l}1077 \\
(65.1)\end{array}$ & $\begin{array}{l}547 \\
(67.0)\end{array}$ & $\begin{array}{l}530 \\
(63.2)\end{array}$ & \\
\hline Father & $\begin{array}{l}578 \\
(34.9)\end{array}$ & $\begin{array}{l}269 \\
(33.0)\end{array}$ & $\begin{array}{l}309 \\
(36.8)\end{array}$ & \\
\hline $\begin{array}{l}\text { Respondent for questions about children's emotional } \\
\text { responses and lifestyle (if children } \geq 10 \text { years old) }\end{array}$ & & & & 0.283 \\
\hline Parent & $\begin{array}{l}491 \\
(54.4)\end{array}$ & $\begin{array}{l}247 \\
(56.3)\end{array}$ & $\begin{array}{l}244 \\
(52.7)\end{array}$ & \\
\hline Children & $\begin{array}{l}411 \\
(45.6)\end{array}$ & $\begin{array}{l}192 \\
(43.7)\end{array}$ & $\begin{array}{l}219 \\
(47.3)\end{array}$ & \\
\hline \multicolumn{5}{|c|}{ * $\mathrm{P}$ value are from Chi square or fisher's exact tests (when applicable). } \\
\hline
\end{tabular}


During the COVID-19 epidemic, 76.7\% (626/816) in Wuhan and 84.7\% (711/839) of children in Shanghai frequently (always, usually or sometimes) asked about the epidemic actively (Table 2 Panel A). A majority of parents explained about the epidemic to their children in Wuhan $(93.0 \%, 780 / 839)$ and Shanghai $(83.8 \%, 684 / 816)$. Nearly half the parents $(42.5 \%, 347 / 816$ in Wuhan and 49.2\%, 413/839 in Shanghai) expressed negative emotions or discussed the epidemic in the presence of their children. Parents generally reported being more patient towards their children than normal $(51.0 \%, 416 / 816$ in Wuhan; $56.6 \%, 475 / 839$ in Shanghai), but there were some parents who reported becoming more irritable during the epidemic (18.0\%, 147/816 in Wuhan and 13.2\%, 111/839 in Shanghai). More than half the parents (435/816, 53.3\% in Wuhan and $511 / 839,60.9 \%$ in Shanghai) reported better parent-child closeness during the epidemic than normal. 
Table 2

Parenting and children's emotional responses during the COVID-19 epidemic in Wuhan and Shanghai, $n$ (\%)

\begin{tabular}{|c|c|c|c|c|}
\hline & $\begin{array}{l}\text { Total sample } \\
(n=1655)\end{array}$ & $\begin{array}{l}\text { Wuhan } \\
(\mathrm{n}= \\
816)\end{array}$ & $\begin{array}{l}\text { Shanghai } \\
(n=839)\end{array}$ & $\begin{array}{l}\text { P- } \\
\text { value* }\end{array}$ \\
\hline \multicolumn{5}{|l|}{ Panel A: Parenting } \\
\hline Children actively asked about the epidemic & & & & $<.001$ \\
\hline Always & $303(18.3)$ & $\begin{array}{l}145 \\
(17.8)\end{array}$ & $\begin{array}{l}158 \\
(18.8)\end{array}$ & \\
\hline Usual & $565(34.1)$ & $\begin{array}{l}269 \\
(33.0)\end{array}$ & $\begin{array}{l}296 \\
(35.3)\end{array}$ & \\
\hline Sometimes & $469(28.3)$ & $\begin{array}{l}212 \\
(26.0)\end{array}$ & $\begin{array}{l}257 \\
(30.6)\end{array}$ & \\
\hline Rare & $222(13.4)$ & $\begin{array}{l}142 \\
(17.4)\end{array}$ & $80(9.5)$ & \\
\hline Never & $96(5.8)$ & $\begin{array}{l}48 \\
(5.9)\end{array}$ & $48(5.7)$ & \\
\hline Parent explained the epidemic to children & & & & $\begin{array}{l}<.001 \\
0.01\end{array}$ \\
\hline Always & $566(34.2)$ & $\begin{array}{l}262 \\
(32.1)\end{array}$ & $\begin{array}{l}304 \\
(36.2)\end{array}$ & \\
\hline Usual & $580(35.1)$ & $\begin{array}{l}274 \\
(33.6)\end{array}$ & $\begin{array}{l}306 \\
(36.5)\end{array}$ & \\
\hline Sometimes & $318(19.2)$ & $\begin{array}{l}148 \\
(18.1)\end{array}$ & $\begin{array}{l}170 \\
(20.3)\end{array}$ & \\
\hline Rare & $183(11.1)$ & $\begin{array}{l}129 \\
(15.8)\end{array}$ & $54(6.4)$ & \\
\hline Never & $8(0.5)$ & $3(0.4)$ & $5(0.6)$ & \\
\hline $\begin{array}{l}\text { Parent expressed negative sentiments or discussed } \\
\text { the epidemic in their child's presence }\end{array}$ & & & & 0.001 \\
\hline Always & $130(7.9)$ & $\begin{array}{l}73 \\
(9.0)\end{array}$ & $57(6.8)$ & \\
\hline
\end{tabular}

\footnotetext{
* $P$ value are from Chi square or fisher's exact tests (when applicable).

\# One's SDQ-Emotional Symptoms Score $>4$ for parent version or $>6$ for child version was considered to have substantial risk of clinically significant emotional problems.
} 


\begin{tabular}{|c|c|c|c|c|}
\hline & $\begin{array}{l}\text { Total sample } \\
(n=1655)\end{array}$ & $\begin{array}{l}\text { Wuhan } \\
(n= \\
816)\end{array}$ & $\begin{array}{l}\text { Shanghai } \\
(n=839)\end{array}$ & $\begin{array}{l}\text { P- } \\
\text { value* }\end{array}$ \\
\hline Usually & $217(13.1)$ & $\begin{array}{l}88 \\
(10.8)\end{array}$ & $\begin{array}{l}129 \\
(15.4)\end{array}$ & \\
\hline Sometimes & $413(25.0)$ & $\begin{array}{l}186 \\
(22.8)\end{array}$ & $\begin{array}{l}227 \\
(27.1)\end{array}$ & \\
\hline Rare & $541(32.7)$ & $\begin{array}{l}269 \\
(33.0)\end{array}$ & $\begin{array}{l}272 \\
(32.4)\end{array}$ & \\
\hline Never & $354(21.4)$ & $\begin{array}{l}200 \\
(24.5)\end{array}$ & $\begin{array}{l}154 \\
(18.4)\end{array}$ & \\
\hline Parent's attitude towards children & & & & 0.013 \\
\hline More irritable & $258(15.6)$ & $\begin{array}{l}147 \\
(18.0)\end{array}$ & $\begin{array}{l}111 \\
(13.2)\end{array}$ & \\
\hline Unchanged & $506(30.6)$ & $\begin{array}{l}253 \\
(31.0)\end{array}$ & $\begin{array}{l}253 \\
(30.2)\end{array}$ & \\
\hline More patient & $891(53.8)$ & $\begin{array}{l}416 \\
(51.0)\end{array}$ & $\begin{array}{l}475 \\
(56.6)\end{array}$ & \\
\hline Parent-child closeness & & & & 0.008 \\
\hline Worse & $177(10.7)$ & $\begin{array}{l}95 \\
(11.6)\end{array}$ & $82(9.8)$ & \\
\hline Unchanged & $532(32.2)$ & $\begin{array}{l}286 \\
(35.1)\end{array}$ & $\begin{array}{l}246 \\
(29.3)\end{array}$ & \\
\hline Better & $946(57.2)$ & $\begin{array}{l}435 \\
(53.3)\end{array}$ & $\begin{array}{l}511 \\
(60.9)\end{array}$ & \\
\hline \multicolumn{5}{|l|}{ Panel B: Children's emotional responses } \\
\hline $\begin{array}{l}\text { Substantial risk of clinically significant emotional } \\
\text { problems }{ }^{\#}\end{array}$ & $163(9.9)$ & $\begin{array}{l}65 \\
(8.0)\end{array}$ & $98(11.7)$ & 0.011 \\
\hline Having stress symptoms & $812(49.1)$ & $\begin{array}{l}322 \\
(39.5)\end{array}$ & $\begin{array}{l}490 \\
(58.4)\end{array}$ & $<.001$ \\
\hline \multicolumn{5}{|c|}{ * $P$ value are from Chi square or fisher's exact tests (when applicable). } \\
\hline
\end{tabular}

A small proportion of children (8.0\%,65/816 in Wuhan and 11.7\%, 98/839 in Shanghai) were categorized as having a substantial risk of clinically significant emotional problems (Table 2 Panel B). A large proportion of children $(39.5 \%, 322 / 816$ in Wuhan and $58.4 \%, 490 / 839$ in Shanghai) reportedly had 
symptoms of stress such as aggressive or stubborn behavior and tantrums or meltdowns during the epidemic.

Figure 2 presents the comparisons of children's lifestyle before and during the COVID-19 epidemic. About half the children went outside during the COVID-19 epidemic in Wuhan $(41.3 \%, 337 / 816)$ and Shanghai $(52.3 \%, 439 / 839)$. The proportions of children with unhealthy lifestyles were higher during than before the epidemic in Wuhan and Shanghai.

\subsection{Inequity in parenting, children's emotional responses and lifestyle changes during the COVID-19 epidemic}

As shown in Table 3, parents with lower educational attainment had more frequent communication about the epidemic with their children than parents with a higher educational attainment. Parents with lower educational attainment, however, had a more irritable attitude towards their children and worse closeness with children during the epidemic. Children of parents with lower educational attainment were more likely to be at risk of clinically significant emotional problems and stress symptoms, but less likely to avoid going outside during the epidemic. No significant differences were found for screen time, exercise and sleep quality for children by their parents' educational levels. Similar results were also observed for parents stratified by family economic status and employment status (Appendix A Table A1). 
Table 3

Equity (ratio between comparison groups) in parenting and children's emotion and lifestyle responses during the COVID-19 epidemic

\begin{tabular}{|c|c|c|c|c|}
\hline & \multicolumn{4}{|c|}{$\begin{array}{l}\text { Ratio between low- and high- } \\
\text { education groups }\end{array}$} \\
\hline & Wuhan & value & Shanghai & $\begin{array}{l}P \\
\text { value }\end{array}$ \\
\hline \multicolumn{5}{|l|}{ Panel A: Parenting } \\
\hline $\begin{array}{l}\text { Children actively asked about the epidemic (Always, usually } \\
\text { or sometimes) }\end{array}$ & 1.10 & 0.023 & 1.06 & 0.086 \\
\hline $\begin{array}{l}\text { Parent explained the epidemic to children (Always, usually } \\
\text { or sometimes) }\end{array}$ & 1.11 & 0.003 & 1.02 & 0.335 \\
\hline $\begin{array}{l}\text { Parent expressed negative sentiments or discussed the } \\
\text { epidemic in their child's presence (Always, usually or } \\
\text { sometimes) }\end{array}$ & 1.31 & 0.002 & 1.27 & 0.001 \\
\hline More irritable attitude towards children & 2.60 & $<.001$ & 1.83 & 0.001 \\
\hline Worse parent-child closeness & 2.74 & $<001$ & 3.05 & $\begin{array}{l}< \\
0.001\end{array}$ \\
\hline \multicolumn{5}{|l|}{ Panel B: Children's emotional responses } \\
\hline $\begin{array}{l}\text { Substantial risk of clinically significant emotional } \\
\text { problems } \#\end{array}$ & 1.86 & 0.009 & 2.00 & $<.001$ \\
\hline Having stress symptoms & 1.09 & 0.402 & 1.17 & 0.013 \\
\hline \multicolumn{5}{|l|}{$\begin{array}{l}\text { Panel C: Children's lifestyle changes during the epidemic } \\
\text { compared with normally }\end{array}$} \\
\hline Going outside during the epidemic & 1.28 & 0.005 & 1.25 & 0.002 \\
\hline More screen time per day & 1.18 & 0.131 & 0.94 & 0.545 \\
\hline Less exercise per week or per day & 1.19 & 0.081 & 0.85 & 0.132 \\
\hline Sleep less at night & 1.24 & 0.163 & 0.87 & 0.397 \\
\hline $\begin{array}{l}\text { More often had difficulty getting sleep or woke up > twice } \\
\text { per night }\end{array}$ & 1.25 & 0.054 & 0.77 & 0.054 \\
\hline \multicolumn{5}{|c|}{$\begin{array}{l}\text { Note. }{ }^{*} \text { Ratios generated by dividing the proportion of the group with three years college or below by } \\
\text { the group with bachelor or above. The ratios indicate the gap between the comparison group - where } 1 \\
\text { implies no gap, and P value are from Chi square or fisher's exact tests (when applicable). }\end{array}$} \\
\hline
\end{tabular}




\subsection{Association between parenting and children's emotional and lifestyle changes during the COVID-19 epidemic}

Table 4 presents associations between parenting characteristics and children's emotional and lifestyle changes during the epidemic using multivariate logistic regressions. After controlling for child and family characteristics, children who frequently enquired about the epidemic and whose parents frequently discussed about the epidemic were more likely to have stress symptoms and an unhealthy lifestyle (more screen time, less sleep and worse sleep quality). Children whose parents frequently expressed negative emotions or discussed about the epidemic in their presence (compared to those who did not express or discuss about the epidemic) had a significantly higher odds of having abnormal emotions ( $A O R=2.64$; $95 \% \mathrm{Cl}=1.68-4.12)$, stress symptoms $(\mathrm{AOR}=2.62 ; 95 \% \mathrm{Cl}=2.08-3.30)$, and worse sleep quality $(\mathrm{AOR}=$ $1.34 ; 95 \% \mathrm{Cl}=1.06-1.71)$ during the epidemic.

In addition, children with irritable parents (compared with children who had parents with either unchanged or better attitudes) had a significantly higher odds of having abnormal emotions (AOR $=2.24 ; 95 \% \mathrm{Cl}=$ $1.42-3.55)$, stress symptoms $(A O R=1.93 ; 95 \% \mathrm{Cl}=1.33-2.81)$, more screen time $(A O R=1.50 ; 95 \% \mathrm{Cl}=$ $1.08-2.09)$, less sleep $(A O R=1.72 ; 95 \% \mathrm{Cl}=1.17-2.51)$, and worse sleep quality $(A O R=1.50 ; 95 \% \mathrm{Cl}=$ 1.07-2.10) during the epidemic. Worse parent-child closeness during the epidemic was also positively associated with children having abnormal emotions ( $A O R=2.93 ; 95 \% \mathrm{Cl}=1.80-4.79$ ), less exercise (AOR $=1.60 ; 95 \% \mathrm{Cl}=1.09-2.35)$, and worse sleep quality $(\mathrm{AOR}=2.36 ; 95 \% \mathrm{Cl}=1.59-3.49)$ during the epidemic . 
Table 4

Associations between parenting and children's emotion and lifestyle responses to the COVID-19 epidemic, multivariate logistic regression.

\begin{tabular}{|c|c|c|c|c|c|c|}
\hline \multirow[t]{2}{*}{ Parenting variables } & \multicolumn{2}{|c|}{ Emotion and stress } & \multicolumn{4}{|c|}{ Lifestyle } \\
\hline & $\begin{array}{l}\text { Abnormal } \\
\text { emotions }\end{array}$ & $\begin{array}{l}\text { Stress } \\
\text { symptoms }\end{array}$ & $\begin{array}{l}\text { More } \\
\text { screen } \\
\text { time }\end{array}$ & $\begin{array}{l}\text { Less } \\
\text { exercise }\end{array}$ & $\begin{array}{l}\text { Sleep } \\
\text { less }\end{array}$ & $\begin{array}{l}\text { Worse } \\
\text { sleep } \\
\text { quality }\end{array}$ \\
\hline $\begin{array}{l}\text { Children actively asked about } \\
\text { the epidemic }\end{array}$ & 2.04 & $1.46^{\star}$ & 0.93 & 1.00 & $0.64^{*}$ & 1.31 \\
\hline $\begin{array}{l}\text { (always, usually or sometimes } \\
\text { VS rare or never) }\end{array}$ & $\begin{array}{l}(0.93- \\
4.49)\end{array}$ & $\begin{array}{l}(1.04- \\
2.06)\end{array}$ & $\begin{array}{l}(0.67- \\
1.29)\end{array}$ & $\begin{array}{l}(0.73- \\
1.38)\end{array}$ & $\begin{array}{l}(0.44- \\
0.94)\end{array}$ & $\begin{array}{l}(0.90- \\
1.90)\end{array}$ \\
\hline $\begin{array}{l}\text { Parent explained the epidemic } \\
\text { to children }\end{array}$ & 0.98 & $2.87 \star \star$ & $1.68^{\star}$ & 1.36 & $2.52^{\star \star}$ & $2.45^{\star \star}$ \\
\hline $\begin{array}{l}\text { (always, usually or sometimes } \\
\text { VS rare or never) }\end{array}$ & $\begin{array}{l}(0.39- \\
2.48)\end{array}$ & $\begin{array}{l}(1.80- \\
4.58)\end{array}$ & $\begin{array}{l}(1.12- \\
2.54)\end{array}$ & $\begin{array}{l}(0.92- \\
2.00)\end{array}$ & $\begin{array}{l}(1.48- \\
4.30)\end{array}$ & $\begin{array}{l}(1.49- \\
4.01)\end{array}$ \\
\hline $\begin{array}{l}\text { Parent expressed negative } \\
\text { sentiments or discussed the } \\
\text { epidemic in their child's } \\
\text { presence }\end{array}$ & $2.64 * \star$ & $2.62^{\star \star}$ & 1.09 & 1.11 & 1.14 & $1.34^{\star}$ \\
\hline $\begin{array}{l}\text { (always, usually or sometimes } \\
\text { VS rare or never) }\end{array}$ & $\begin{array}{l}(1.68- \\
4.12)\end{array}$ & $\begin{array}{l}(2.08- \\
3.30)\end{array}$ & $\begin{array}{l}(0.87- \\
1.36)\end{array}$ & $\begin{array}{l}(0.89- \\
1.39)\end{array}$ & $\begin{array}{l}(0.87- \\
1.49)\end{array}$ & $\begin{array}{l}(1.06- \\
1.71)\end{array}$ \\
\hline Attitude towards children & $2.24 * *$ & $1.93^{\star \star}$ & $1.50 *$ & 1.01 & $1.72^{\star \star}$ & $1.50 *$ \\
\hline $\begin{array}{l}\text { (more irritable VS more patient } \\
\text { or unchanged) }\end{array}$ & $\begin{array}{l}(1.42- \\
3.55)\end{array}$ & $\begin{array}{l}(1.33- \\
2.81)\end{array}$ & $\begin{array}{l}(1.08- \\
2.09)\end{array}$ & $\begin{array}{l}(0.72- \\
1.40)\end{array}$ & $\begin{array}{l}(1.17- \\
2.51)\end{array}$ & $\begin{array}{l}(1.07- \\
2.10)\end{array}$ \\
\hline Parent-child closeness & $2.93^{\star \star}$ & 1.18 & 1.02 & $1.60 *$ & 0.77 & $2.36^{\star \star}$ \\
\hline (worse VS better or unchanged) & $\begin{array}{l}(1.80- \\
4.79)\end{array}$ & $\begin{array}{l}(0.76- \\
1.83)\end{array}$ & $\begin{array}{l}(0.69- \\
1.52)\end{array}$ & $\begin{array}{l}(1.09- \\
2.35)\end{array}$ & $\begin{array}{l}(0.48- \\
1.24)\end{array}$ & $\begin{array}{l}(1.59- \\
3.49)\end{array}$ \\
\hline Observations & 1,655 & 1,655 & 1,655 & 1,655 & 1,655 & 1,655 \\
\hline $\begin{array}{l}\text { Note. Adjusted odds ratios with } \\
\text { regressions. We controlled for ch } \\
\text { during the epidemic), family cha } \\
\text { cases in neighborhood), respon }\end{array}$ & $\begin{array}{l}\text { confidenc } \\
\text { en charac }\end{array}$ & $\begin{array}{l}\text { ntervals we } \\
\text { stics (gend }\end{array}$ & obtaine & rom mul & $\begin{array}{l}\text { ariate lo } \\
\text { or going }\end{array}$ & $\begin{array}{l}\text { utic } \\
\text { utside } \\
\text { VID-19 } \\
5 .\end{array}$ \\
\hline
\end{tabular}

\section{Discussion}

Our study investigated parenting and children's emotional and lifestyle responses to the COVID-19 epidemic in China. More than $80 \%$ of parents or children frequently communicated about the COVID-19 epidemic, and more than $10 \%$ of parents had a more irritable attitude and reported worse parent-child closeness during the epidemic. Around $10 \%$ of children were at a risk of clinically significant emotional problems, and about half of the children had stress symptoms during the epidemic. Our study also found 
a high prevalence (30\% 80\%) of unhealthy lifestyles both at normal times (before the epidemic) and during the epidemic, with a higher prevalence during the epidemic. Differing emotional and lifestyle responses were observed among children from socially disadvantaged families, making them more vulnerable to the epidemic. Children whose parents frequently communicated about COVID-19, or were more irritable, or who experienced worse closeness with their parents were more likely to have internalized emotional problems, stress symptoms, and worse lifestyles during the COVID-19 epidemic.

Our survey found a high prevalence of inactive children even during normal times (before the epidemic), concurring with the findings of earlier studies. In a pooled analysis of 298 population-based surveys, the overall prevalence of insufficient physical activity was $84.3 \%$ among Chinese adolescents aged 1117 years in 2016 [23], and in another study with a sample of 131,859 Chinese students aged 7-19 years, about $35 \%$ of children did not meet the recommended screen viewing time [11]. Moreover, we found that screen viewing time and physical activity levels worsened during the epidemic (compared to normal times) in both Wuhan and Shanghai. This finding highlights potential challenges to China achieving the WHO target of a $15 \%$ relative reduction in insufficient physical activity for children by 2030 due to the COVID-19 epidemic [24]. Furthermore, lesser hours of sleep and worse sleep quality were observed among children during the epidemic; the prevalence of children and adolescents sleeping less than 8 hours increased from $34 \%$ normally to $54 \%$ during the epidemic. Children's sleep, physical activity and screen time are often found interacted, where more screen time and less time outdoors are known to be adversely associated with sleep outcomes $[25,26]$. Therefore, a multi-tiered approach involving the society, school and family is required to promote better mental and physical health for children and adolescents during the epidemic and similar public health emergencies [27].

In our study, parenting characteristics were observed to play an important role in children's emotional and lifestyle responses to the COVID-19 epidemic. Frequent communication about the epidemic between parents and children, having irritable parents and worse parent-child closeness were observed to be associated with an increased odd of emotional problems and unhealthy lifestyles among children. There was an increased spread of misinformation and rumors about the epidemic, and excessive exposure to negative information may increase fears and anxieties for both parents and children. Our findings concur with the view that parents who lack appropriate skill or guidance to help themselves and their children face greater adversity during a public health emergency. A previous study also found similar results for adults; adults who spent more time focusing on the epidemic were more likely to develop anxiety symptoms [28]. Family systems theory suggests that children and adolescents in families are strongly influenced by marital problems and poor parenting [15]. If parents have psychological distress, they are likely to transmit their distress during interactions with their children and create a negative atmosphere which could lead to persistent or delayed-onset distress symptoms [27]. Many studies have illustrated that mental health is worst among children whose caregivers have experienced negative mental health outcomes from disasters [29-31]. Therefore, health education should be provided to parents to improve their knowledge about the epidemic and reinforce good parenting skills to improve the quality of communication between parents and children. Parents should be educated with scientific information to 
generate awareness about the importance of healthy lifestyles such as ongoing physical activity and reduced screen time especially during the epidemic.

Our study also shows that children from socially-disadvantaged families have worse parenting, emotional and lifestyle responses to the epidemic than those from advantaged families. Parents who are socially disadvantaged (in terms of education, employment or economic status) may have lower health literacy and awareness about their own or their child's health risks; and they may not be able to recognize negative changes in their child's emotional health and lifestyles and provide timely support to their children [32,33]. In addition, socially disadvantaged parents may also be more likely to suffer psychological stresses and other health problems themselves, especially during the epidemic [34,35]. A previous study observed that parents who had not returned to work due to the epidemic were more likely to experience higher stress and anxiety regarding job continuity and financial security [25]. Highly stressed parents may in turn be more irritable towards their children and more likely to express negative emotions in their child's presence, which may lead to more frequent emotional and lifestyle problems among their children. Therefore, it is vital to deliver interventions targeting parents from such disadvantaged groups, providing them appropriate health education and psychological services to decrease the disproportionate emotional and behavioral responses their children face during the COVID19 epidemic.

Our study had a few limitations. First, there may be a degree of recall bias as we aimed to investigate changes in children's lifestyle outcomes during the COVID-19 epidemic compared to the previous winter break (based on parent recall). Second, most respondents were parents instead of the children themselves, which may lead to reporting bias. Third, our results may be affected by selection bias from the use of an online survey methodology. We sent multiple invitations to respondents who did not initially respond to our survey to mitigate this bias. Finally, this was a cross-sectional study, and there is a need for follow-up studies on the emotional and lifestyle changes of children and adolescents during public health emergencies such as the COVID-19 epidemic to validate our findings.

\section{Conclusion}

During the COVID-19 epidemic, about half (49\%) of the children had stress symptoms and around $10 \%$ of children had a risk of clinically significant emotional problems. Children were also observed to have higher screen time, lesser physical activity and worse sleep during the epidemic than before it. Frequent parent-child communication about the epidemic, having irritable parents and parents who reported worse parent-child closeness were associated with higher probabilities of children having emotional problems, stress symptoms and detrimental lifestyle changes. Disproportionate emotional and lifestyle responses to the epidemic were observed among children from socially disadvantaged families. To improve children's emotional and lifestyle responses to the epidemic, health education should be provided to parents to improve their knowledge about the epidemic and on healthy lifestyles for their children. It is also important to promote better parenting to improve the quality of communication between parents and children both during and after the epidemic, especially for disadvantaged families. 


\section{Declarations}

\section{Acknowledgements}

We are thankful to Hao Jiang and Biao Wang from the School of Public Health, Fudan University for helping with data collection.

\section{Consent for publication.}

Not applicable.

\section{Availability of data and materials.}

The datasets used and/or analyzed during the current study are available from the corresponding author on reasonable request.

\section{Contributors}

$\mathrm{HY}$ and ZH designed the study. ZH and QL developed the survey questionnaire, collected data. FD analyzed data. ZH, FD and LH interpreted results and wrote the manuscript. ZH, LH, Mark Francis, Mark Forshaw, KW and LS critically revised the manuscript for important intellectual content. All authors approved the final manuscript.

\section{Ethical approval}

The study was approved by the institutional review board at School of Public Health, Fudan University (IRB\#2020-01-0801-S). We received written informed consent from at least one of parent or guardian for all children participating in the survey. All methods were performed in accordance with the relevant guidelines and regulations proposed by the institutional review board at School of Public Health, Fudan University.

\section{Funding}

This work was supported by the National Natural Science Foundation of China (No. 71874034); the National Science Fund for Distinguished Young Scholars (No. 81525023); National Science and Technology Major Project of China (No. 2018ZX10201001-010, No. 2017ZX10103009-005, No. 2018ZX10713001-007). The funders of the study had no role in study design, data collection, data analysis, data interpretation or writing of the report. 


\section{Competing interests}

HY has received research funding from Sanofi Pasteur, GlaxoSmithKline, Yichang HEC Changjiang Pharmaceutical Company and Shanghai Roche Pharmaceutical Company. None of that research funding is related to COVID-19.

\section{References}

1. Zhou F, Yu T, Du R, et al. Clinical course and risk factors for mortality of adult inpatients with COVID19 in Wuhan, China: a retrospective cohort study. Lancet 2020;395(10229):1054-1062. https://doi.org/10.1016/S0140-6736(20)30566-3.

2. Parmet WE, Sinha MS. Covid-19 - The Law and Limits of Quarantine. N Engl J Med 2020;382(15):e28. https://doi.org/10.1056/NEJMp2004211.

3. Qin F, Song Y, Nassis GP, et al. Physical Activity, Screen Time, and Emotional Well-Being during the 2019 Novel Coronavirus Outbreak in China. Int J Environ Res Public Health 2020;17(14):5170. https://doi.org/10.3390/ijerph17145170.

4. Zhu Z, Liu Q, Jiang X, et al. The psychological status of people affected by the COVID-19 outbreak in China. J Psychiatr Res 2020;129:1-7. https://doi.org/10.1016/j.jpsychires.2020.05.026.

5. Wang G, Zhang Y, Zhao J, et al. Mitigate the effects of home confinement on children during the COVID-19 outbreak. Lancet 2020;395(10228):945-947. https://doi.org/10.1016/S01406736(20)30547-X.

6. Belhadj K E, Koglin U, Petermann F. Emotional and behavioral problems in migrant children and adolescents in Europe: a systematic review. Eur Child Adolesc Psychiatry 2014;23(6), 373-391. https://doi.org/10.1007/s00787-013-0485-8.

7. Luo J, Zou J, Ji M, et al. Emotional and Behavioral Problems Among 3- to 5-Year-Olds Left-Behind Children in Poor Rural Areas of Hunan Province: A Cross-Sectional Study. Int J Environ Res Public Health 2019;16(21):4188. https://doi.org/10.3390/ijerph16214188.

8. Yang Y, Qi Y, Cui Y, et al. Emotional and behavioral problems, social competence and risk factors in 616-year-old students in Beijing, China. PLoS One 2019;14(10):e0223970.

https://doi.org/10.1371/journal.pone.0223970.

9. World Health Organization. WHO Guidelines on physical activity and sedentary behaviour for children and adolescents, adults and older adults. Available at: https://www.who.int/docs/defaultsource/physical-activity/call-for-consultation/draft-guideline-on-physical-activity-and-sedentraybehaviour.pdf?sfvrsn=ddf523d5_4. Accessed October 19, 2020.

10. Hirshkowitz M, Whiton K, Albert SM, et al. National Sleep Foundation's updated sleep duration recommendations: final report. Sleep Health 2015;1(4):233-243. https://doi.org/10.1016/j.sleh.2015.10.004. 
11. Zhu Z, Tang Y, Zhuang J, et al. Physical activity, screen viewing time, and overweight/obesity among Chinese children and adolescents: an update from the 2017 physical activity and fitness in China-the youth study. BMC Public Health 2019;19(1):197. https://doi.org/10.1186/s12889-019-6515-9.

12. Armitage R, Nellums LB. Considering inequalities in the school closure response to COVID-19. Lancet Glob Health 2020;8(5):e644. https://doi.org/10.1016/S2214-109X(20)30116-9.

13. Qi M, Zhou SJ, Guo ZC, et al. The Effect of Social Support on Mental Health in Chinese Adolescents During the Outbreak of COVID-19. J Adolesc Health 2020;67(4):514-518. https://doi.org/10.1016/j.jadohealth.2020.07.001.

14. Zhang W, Liu H, Jiang $X$, et al. A longitudinal study of posttraumatic stress disorder symptoms and its relationship with coping skill and locus of control in adolescents after an earthquake in China. PLoS One 2014;9(2):e88263. https://doi.org/10.1371/journal.pone.0088263.

15. Miller IW, Ryan CE, Keitner GI, et al. The McMaster Approach to Families: theory, assessment, treatment and research. Journal of Family Therapy 2000;22(2), 168-189. https://doi.org/10.1111/1467-6427.00145.

16. Fegert J M, Vitiello B, Plener P L, Clemens V. Challenges and burden of the Coronavirus 2019 (COVID19) pandemic for child and adolescent mental health: a narrative review to highlight clinical and research needs in the acute phase and the long return to normality. Child and Adolescent Psychiatry and Mental Health 2020;14:20. https://doi.org/10.1186/s13034-020-00329-3.

17. Rubin GJ, Amlôt R, Page L, Wessely S. Public perceptions, anxiety, and behaviour change in relation to the swine flu outbreak: cross sectional telephone survey. BMJ 2009;339:b2651. https://doi.org/10.1136/bmj.b2651.

18. Sedgwick P. Proportional quota sampling. British Medical Journal 2012;345(e6336).

19. National Bureau of Statistics of China, 2010. Tabulation on the 2010 publication census of the People's Republic of China. Available at: http://www.stats.gov.cn/tjsj/pcsj/rkpc/6rp/indexch.htm. Accessed November 11, 2020.

20. Australian Mental Health Outcomes and Classification Network. Mental Health National Outcomes and Casemix Collection: Overview of Clinician-Rated and Consumer Self-Report Measures. Available at: https://www.amhocn.org/sites/default/files/publication_files/mhnocc_overview_doha.pdf. Accessed November 11, 2020.

21. Du Y, Kou J, Coghill D. The validity, reliability and normative scores of the parent, teacher and self report versions of the Strengths and Difficulties Questionnaire in China. Child Adolesc Psychiatry Ment Health 2008;2(1), 8. https://doi.org/10.1186/1753-2000-2-8.

22. Bruni O, Ottaviano S, Guidetti V, et al. The Sleep Disturbance Scale for Children (SDSC). Construction and validation of an instrument to evaluate sleep disturbances in childhood and adolescence. $J$ Sleep Res 1996;5(4):251 - 61. https://doi.org/10.1111/j.1365-2869.1996.00251.x.

23. Guthold R, Stevens G A, Riley L M, Bull F C. Global trends in insufficient physical activity among adolescents: a pooled analysis of 298 population-based surveys with 1.6 million participants. Lancet Child Adolesc Health 2020;4(1):23-35. https://doi.org/10.1016/S2352-4642(19)30323-2. 
24. World Health Organization. Global action plan on physical activity 2018-2030: more active people for a healthier world. Available at: https://www.who.int/ncds/prevention/physical-activity/globalaction-plan-2018-2030/en/. Accessed November 11, 2020.

25. Altena E, Baglioni C, Espie CA, et al. Dealing with sleep problems during home confinement due to the COVID-19 outbreak: Practical recommendations from a task force of the European CBT-I Academy. J Sleep Res 2020;29(4):e13052. https://doi.org/10.1111/jsr.13052.

26. Hale L, Guan S. Screen time and sleep among school-aged children and adolescents: a systematic literature review. Sleep Med Rev 2015;21:50 - 8. https://doi.org/10.1016/j.smrv.2014.07.007.

27. Zhou X. Managing psychological distress in children and adolescents following the COVID-19 epidemic: A cooperative approach. Psychol Trauma 2020;12(S1):S76-S78. https://doi.org/10.1037/tra0000754.

28. Huang Y, Zhao N. Generalized anxiety disorder, depressive symptoms and sleep quality during COVID-19 outbreak in China: a web-based cross-sectional survey. Psychiatry Res 2020;288:112954. https://doi.org/10.1016/j.psychres.2020.112954.

29. Kerns CE, Elkins RM, Carpenter AL, et al. Caregiver distress, shared traumatic exposure, and child adjustment among area youth following the 2013 Boston Marathon bombing. J Affect Disord 2014;167:50 - 5. https://doi.org/10.1016/j.jad.2014.05.040.

30. Russell BS, Hutchison M, Tambling R, et al. Initial Challenges of Caregiving During COVID-19: Caregiver Burden, Mental Health, and the Parent-Child Relationship. Child Psychiatry Hum Dev 2020;51(5):671-682. https://doi.org/10.1007/s10578-020-01037-x.

31. Spinelli M, Lionetti F, Pastore M, Fasolo M. Parents' Stress and Children's Psychological Problems in Families Facing the COVID-19 Outbreak in Italy. Front Psychol 2020;11:1713. https://doi.org/10.3389/fpsyg.2020.01713.

32. Lastrucci V, Lorini C, Caini S, et al. Health literacy as a mediator of the relationship between socioeconomic status and health: A cross-sectional study in a population-based sample in Florence. PLoS One. 14(12):e0227007. https://doi.org/10.1371/journal.pone.0227007.

33. Rajah R, Hassali MAA, Murugiah MK. A systematic review of the prevalence of limited health literacy in Southeast Asian countries. Public Health 2019;167:8-15. https://doi.org/10.1016/j.puhe.2018.09.028.

34. Sun $P$, Unger JB, Palmer $P$, et al. Relative income inequality and selected health outcomes in urban Chinese youth. Soc Sci Med 2012;74(1):84-91. https://doi.org/10.1016/j.socscimed.2011.10.010.

35. Kaur M, Chakrapani V, Newtonraj A, et al. Social capital as a mediator of the influence of socioeconomic position on health: Findings from a population-based cross-sectional study in Chandigarh, India. Indian J Public Health 2018;62(4):294-298. https://doi.org/10.4103/ijph.IJPH_274_17.

\section{Figures}




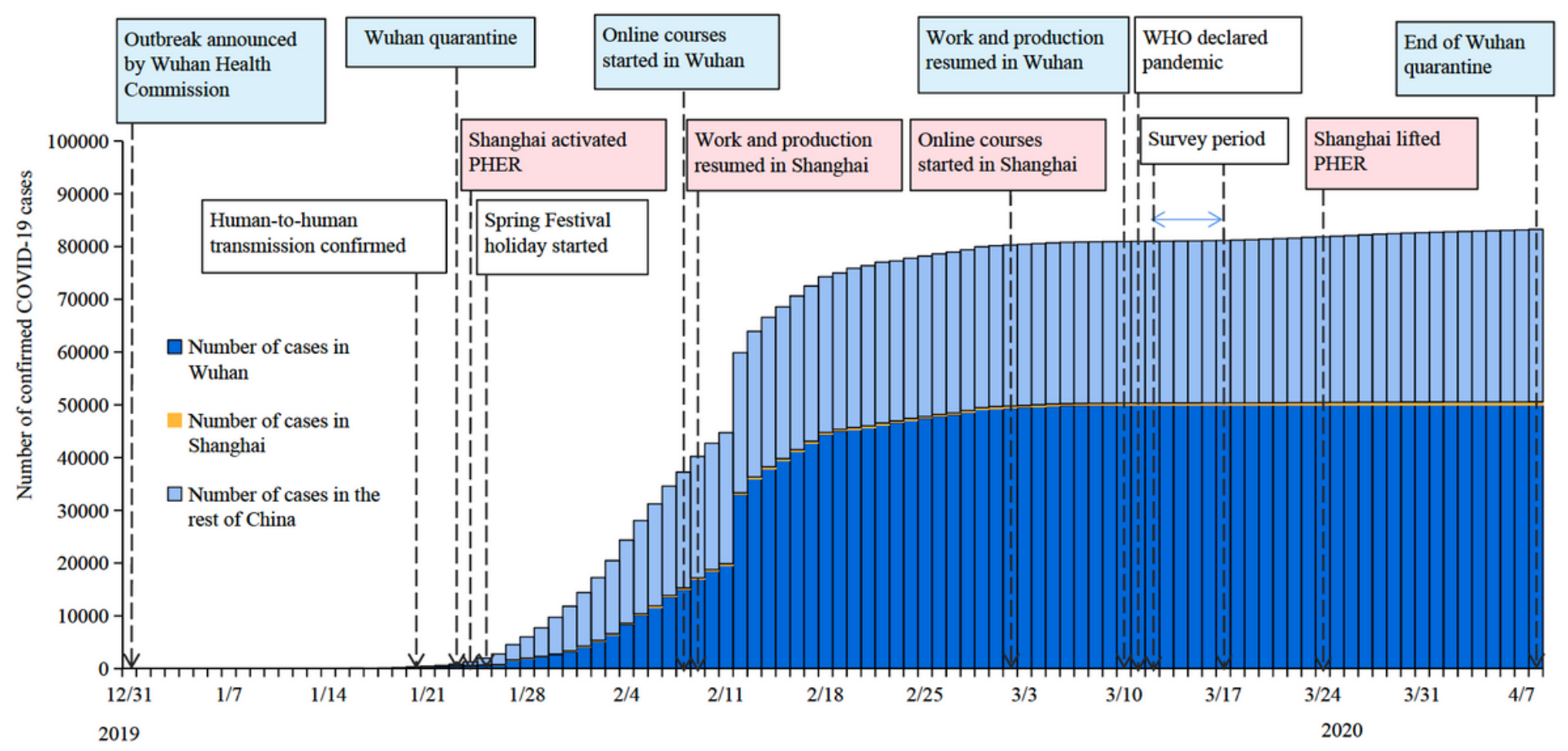

Figure 1

The timeline of the COVID-19 epidemic progression and quarantine measures in Wuhan and Shanghai Note. PHER: public health emergency response.

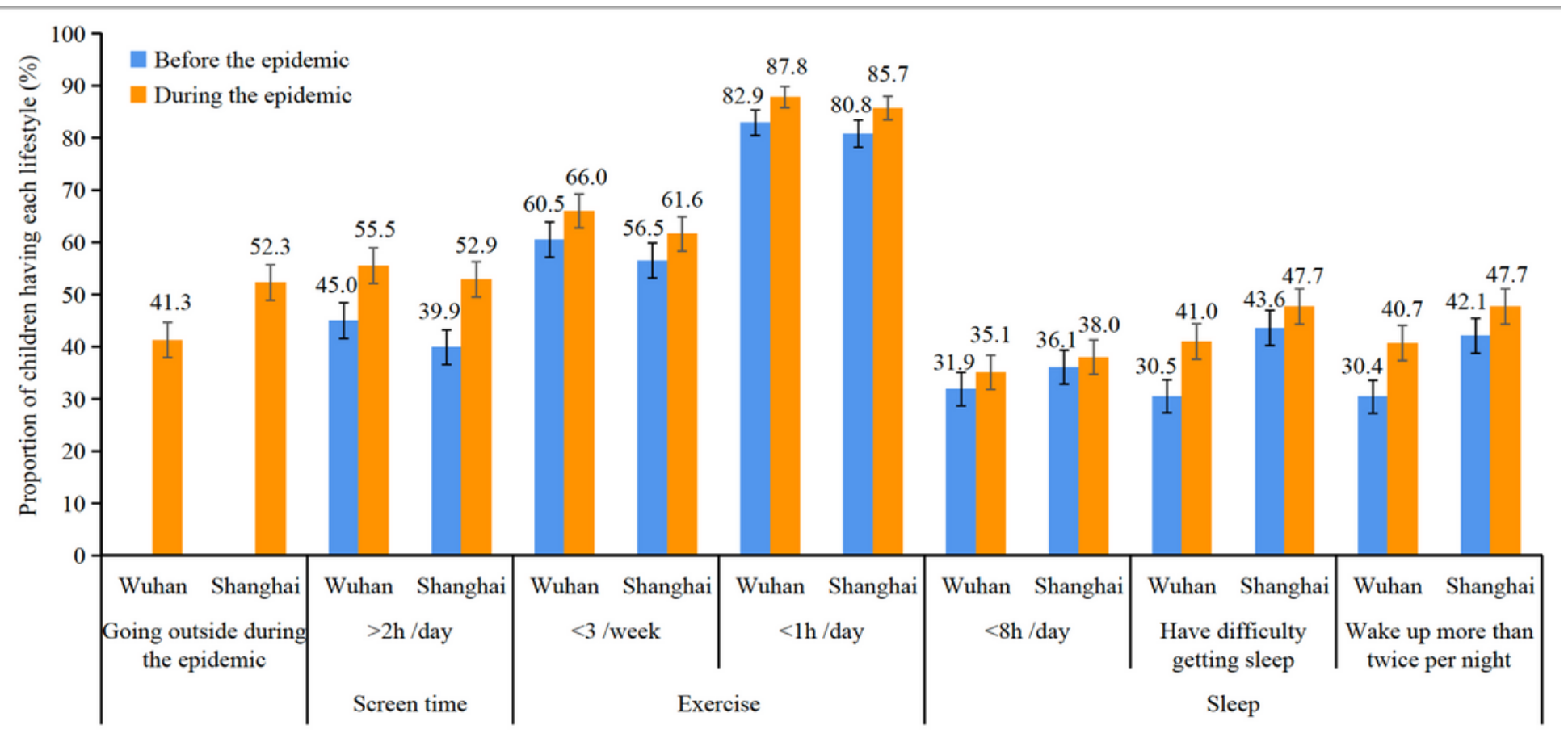

\section{Figure 2}

Comparing the proportions of children having unhealthy lifestyles before and during the COVID-19 epidemic Note. Percentage (\%) and 95\% confidence interval were presented. 


\section{Supplementary Files}

This is a list of supplementary files associated with this preprint. Click to download.

- Appendix.docx 